\title{
Parabolic solutions for the planar $N$-centre problem: multiplicity and scattering
}

\author{
Alberto Boscaggin ${ }^{1}$. Walter Dambrosio ${ }^{1}$. \\ Duccio Papini ${ }^{2}$
}

Received: 13 April 2017 / Accepted: 12 October 2017 / Published online: 20 October 2017

(c) Fondazione Annali di Matematica Pura ed Applicata and Springer-Verlag GmbH Germany 2017

Abstract For the planar $N$-centre problem

$$
\ddot{x}=-\sum_{i=1}^{N} \frac{m_{i}\left(x-c_{i}\right)}{\left|x-c_{i}\right|^{\alpha+2}}, \quad x \in \mathbb{R}^{2} \backslash\left\{c_{1}, \ldots, c_{N}\right\},
$$

where $m_{i}>0$ for $i=1, \ldots, N$ and $\alpha \in[1,2)$, we prove the existence of entire parabolic trajectories, having prescribed asymptotic directions for $t \rightarrow \pm \infty$ and prescribed topological characterization with respect to the set of the centres.

Keywords $N$-centre problem $\cdot$ Parabolic solutions $\cdot$ Scattering

Mathematics Subject Classification 37J45 - 70B05 · 70F16

Work partially supported by the ERC Advanced Grant 2013 No. 339958 Complex Patterns for Strongly Interacting Dynamical Systems-COMPAT, by the PRIN-2012-74FYK7 Grant Variational and perturbative aspects of nonlinear differential problems and by the INDAM-GNAMPA Project Dinamiche complesse per il problema degli $N$-centri.

$凶 \quad$ Duccio Papini

duccio.papini@uniud.it

Alberto Boscaggin

alberto.boscaggin@unito.it

Walter Dambrosio

walter.dambrosio@unito.it

1 Dipartimento di Matematica “Giuseppe Peano”, Università di Torino, Via Carlo Alberto, 10, 10123 Torino, Italy

2 Dipartimento di Scienze Matematiche, Informatiche e Fisiche, Università di Udine, Via delle Scienze, 206, 33100 Udine, Italy 


\section{Introduction and statement of the main result}

The $N$-centre problem is the problem of the motion of a test particle in the attracting field generated by $N$ fixed heavy bodies $c_{1}, \ldots, c_{N}$; in celestial mechanics, it often arises as a simplified version of the restricted circular $(N+1)$-body problem in a rotating frame, when centrifugal and Coriolis' forces are neglected. For $N=1$, of course, it just reduces to the classical Kepler problem, while the case $N=2$ has been solved by Jacobi (see, for instance, [21]). For $N \geq 3$, on the contrary, the problem has been proved to be analytically nonintegrable [5] and, in spite of its simple-looking structure, can indeed exhibit very complicated dynamics (see, among others, $[7,8,13-15,18]$ ).

In this paper we will deal with the planar generalized $N$-centre problem

$$
\ddot{x}=-\sum_{i=1}^{N} \frac{m_{i}\left(x-c_{i}\right)}{\left|x-c_{i}\right|^{\alpha+2}}, \quad x \in \mathbb{R}^{2} \backslash\left\{c_{1}, \ldots, c_{N}\right\},
$$

where $\alpha \in[1,2)$, thus including the classical Newtonian case $\alpha=1$ as a particular case; of course, $m_{i}>0$ for $i=1, \ldots, N$. Notice that the above equation has an Hamiltonian structure, with total energy given by

$$
H(x, \dot{x})=\frac{1}{2}|\dot{x}|^{2}-\sum_{i=1}^{N} \frac{m_{i}}{\alpha\left|x-c_{i}\right|^{\alpha}} .
$$

With this in mind, our aim is to prove the existence of entire parabolic (i.e., zero-energy) solutions to (1) having prescribed asymptotic directions at $\pm \infty$. More precisely, denoting by $\Sigma=\left\{c_{1}, \ldots, c_{N}\right\}$ the set of the centres and naming partition of $\Sigma$ any subset $\mathcal{P} \subset \Sigma$ with $\mathcal{P} \neq \emptyset$ and $\mathcal{P} \neq \Sigma$, our main result reads as follows.

Theorem 1.1 Let $N \geq 2$. For any asymptotic directions $\xi^{-}, \xi^{+} \in \mathbb{S}^{1}$ with $\xi^{-} \neq \xi^{+}$and for any partition $\mathcal{P}$ of $\Sigma$, there exists a self-intersection-free parabolic solution $x: \mathbb{R} \rightarrow \mathbb{R}^{2} \backslash \Sigma$ of (1) satisfying $|x(t)| \rightarrow \infty$ for $t \rightarrow \pm \infty$,

$$
\lim _{t \rightarrow \pm \infty} \frac{x(t)}{|x(t)|}=\xi^{ \pm}
$$

and separating the set $\Sigma$ according to the partition $\mathcal{P}$.

A comment about the statement: by the Jordan Theorem on a sphere, the above parabolic solution divides the plane into two connected components, both unbounded (see for instance [10, Lemma 2.1]); accordingly, the sentence "separating the set $\Sigma$ according to the partition $\mathcal{P}$ " means that two centres lie in the same connected component if and only if they are both in $\mathcal{P}$ or both in $\Sigma \backslash \mathcal{P}$.

Theorem 1.1 has to be interpreted in the context of scattering; indeed, it shows how the presence of two or more centres gives rise to (zero-energy) connections between any pair of asymptotic directions (but different), thus allowing in particular any value for the scattering angle. We stress that the analysis of the zero-energy case seems to be particularly interesting from this point of view; indeed, it is well known that for the central potential $V_{\alpha}(x)=\frac{m}{\alpha|x|^{\alpha}}$ (corresponding to the case $N=1$ in the generalized $N$-centre problem) all parabolic solutions span an angle of $2 \pi /(2-\alpha)$ (see, for instance, [9, Proposition 6.1]). This is in strong contrast with the positive energy case, where all (but one) scattering angles are always achieved; accordingly, it is immediately understood that the possibility of an arbitrary zero-energy scattering angle as in Theorem 1.1 is a genuine consequence of the presence of 
$N \geq 2$ centres and of the interaction of a parabolic solution with them. Incidentally, let us observe that, by collapsing all the centres into a single one, such parabolic solutions converge to the juxtaposition of two rectilinear zero-energy solutions of the $\alpha$-Kepler problem (see Remark 3.3). From this perspective, we can also interpret Theorem 1.1 as a continuationtype result, producing, however, classical solutions starting from generalized ones (the case $\xi^{-}=\xi^{+}$being indeed the only one in which we cannot rule out the presence of collisions).

We refer the reader to $[3,4,12,16,17]$ for interesting investigations, from different point of views, about zero-energy solutions of various problems in celestial mechanics; we notice that, in spite of the differences between the considered models, all these results show the crucial role of parabolic solutions as carriers from different regions of the phase-space, in complete agreement with Theorem 1.1. We also mention that an extensive analysis of the scattering process for the planar $N$-centre problem has already be given in the excellent monograph [14] by Klein and Knauf, dealing, however, only with the Newtonian case $(\alpha=1)$ and with positive energy solutions. The results therein are obtained via a global regularization of the problem, allowing to apply the theory of geodesics on surfaces of negative curvature. It is plausible that some results for the zero-energy case could be derived via a limiting procedure; we stress, however, that our approach is more direct and it allows the study of the generalized problem (1) with $\alpha \in[1,2)$ in a unified way.

For the proof of our result, we combine indeed the variational approach to the construction of topologically non-trivial solutions of the Bolza boundary value problem associated with (1), developed in $[18,19]$, together with a limiting procedure introduced in the recent paper [9], dealing with parabolic solutions of the $N$-centre problem in the three-dimensional space. Both these tools are available when $\alpha \in[1,2)$; it has to be emphasized, however, that the Newtonian case is still more difficult, and indeed requires the use of some (local, LeviCivita type) regularization techniques. We also notice that, while in the spatial case solutions of the (fixed-energy) Bolza problem were found via a min-max argument, thus producing entire solutions with (at least generically) non-trivial Morse index, here minimization of the Maupertuis functional in suitable homotopy classes is enough, thus leading to locally minimal solutions.

As a final comment, we remark that the multiplicity pattern in Theorem 1.1 is a consequence of the result proved in $[18,19]$, providing solutions separating the set of the centres according to any given partition of it. It is likely that the use of more refined arguments, on the lines of $[11,22]$, could lead to solutions in different homotopy classes, allowing for self-intersections and revolutions around the centres; in this way, one should obtain a much richer zero-energy dynamics, including scattering solutions, semi-bounded solutions as well as bounded orbits exhibiting symbolic dynamics. Moreover, one of the reviewers pointed out that it should be possible to obtain infinitely many noncollision parabolic solutions with prescribed asymptotic directions as well as chaotic dynamics by using the results of the very recent paper [6]. All this will be the subject of a future investigation.

\subsection{Plan of the paper}

In Sect. 2 we review the existence of topologically non-trivial parabolic solutions of the Bolza problem, while in Sect. 3 we show how to obtain entire parabolic solutions via a limiting procedure. Actually, we are going to prove that the conclusion of Theorem 1.1 holds true for a larger class of equations of the type

$$
\ddot{x}=\nabla U(x), \quad x \in \mathbb{R}^{2} \backslash \Sigma,
$$


under suitable assumptions on the potential $U \in \mathcal{C}^{\infty}\left(\mathbb{R}^{2} \backslash \Sigma\right)$ which we are going to list here below. First of all, we require

$$
U(x)>0, \quad \text { for every } x \in \mathbb{R}^{2} \backslash \Sigma .
$$

Second, dealing with the behaviour of $U$ near the centres we assume that, for some $\alpha \in[1,2)$,

$$
U(x)=\frac{m_{i}}{\alpha\left|x-c_{i}\right|^{\alpha}}+U_{i}(x), \quad i=1, \ldots, N,
$$

where $m_{i}>0$ and $U_{i}$ is smooth on $\left.\mathbb{R}^{2} \backslash\left(\Sigma \backslash\left\{c_{i}\right\}\right)\right)$. Finally, as for the behaviour of $U$ at infinity, we require that, with the same $\alpha$ as above and some $m>0$,

$$
U(x)=\frac{m}{\alpha|x|^{\alpha}}+W(x),
$$

where, for some $\beta>\alpha / 2+1$,

$$
W(x)=O\left(\frac{1}{|x|^{\beta}}\right) \text { and } \quad \nabla W(x)=O\left(\frac{1}{|x|^{\beta+1}}\right), \quad \text { for }|x| \rightarrow+\infty .
$$

It is easy to verify that the potential

$$
V(x)=\sum_{i=1}^{N} \frac{m_{i}}{\alpha\left|x-c_{i}\right|^{\alpha}},
$$

giving rise to the generalized $N$-centre problem (1), satisfies all the above conditions, with $m=\sum_{i=1}^{N} m_{i}$ and $\beta=\alpha+1$.

\section{Parabolic solutions of the Bolza problem}

In this section we look for solutions of the (free-time) fixed-endpoints problem

$$
\left\{\begin{array}{l}
\ddot{x}=\nabla U(x) \\
x( \pm \omega)=q^{ \pm}
\end{array}\right.
$$

satisfying the zero-energy relation

$$
\frac{1}{2}|\dot{x}|^{2}=U(x)
$$

recall that solutions of (6) satisfying (7) are called parabolic solutions of (6). Motivated by the final application, and in order to make all the discussion more transparent, we assume from the beginning that

$$
\left|q^{-}\right|=\left|q^{+}\right| \quad \text { and } \quad q^{-} \neq q^{+}
$$

also, we suppose that $\left|c_{i}\right|<\left|q^{-}\right|$for $i=1, \ldots, N$, that is, all the centres lie inside the ball centred at the origin and of radius $\left|q^{-}\right|=\left|q^{+}\right|$.

Having in mind a variational approach, we introduce the Maupertuis functional

$$
\mathcal{M}(u)=\int_{-1}^{1}|\dot{u}(t)|^{2} \mathrm{~d} t \int_{-1}^{1} U(u(t)) \mathrm{d} t
$$

defined on the Hilbert manifold

$$
\widehat{\Gamma}=\widehat{\Gamma}_{q^{ \pm}}=\left\{u \in H^{1}\left([-1,1] ; \mathbb{R}^{2} \backslash \Sigma\right): u( \pm 1)=q^{ \pm}\right\}
$$


notice that, in view of (3), it holds that $\mathcal{M}(u) \geq 0$ for any $u \in \widehat{\Gamma}$. As well known (see, for instance, $[1$, Theorem 4.1] and [19, Appendix B]) $\mathcal{M}$ is smooth and any critical point $u \in \widehat{\Gamma}$ satisfies, for $t \in[-1,1]$,

$$
\ddot{u}(t)=\omega^{2} \nabla U(u(t)), \quad \frac{1}{2}|\dot{u}(t)|^{2}-\omega^{2} U(u(t))=0,
$$

where

$$
\omega=\left(\frac{\int_{-1}^{1}|\dot{u}(t)|^{2} \mathrm{~d} t}{2 \int_{-1}^{1} U(u(t)) \mathrm{d} t}\right)^{1 / 2}
$$

Observe that, since $q^{+} \neq q^{-}, u$ is not constant: as a consequence, $\omega>0$ and the function

$$
x(t)=u\left(\frac{t}{\omega}\right), \quad t \in[-\omega, \omega]
$$

is easily seen to be a parabolic solution of $\ddot{x}=\nabla U(x)$ on the interval $[-\omega, \omega]$; moreover, of course, $x( \pm \omega)=q^{ \pm}$.

Following [18,19], multiple critical points of $\mathcal{M}$ can be found by minimizing in suitable homotopy classes. Precisely, write $q^{ \pm}=\left|q^{ \pm}\right| e^{i \theta_{ \pm}}$, for suitable $\theta^{ \pm} \in[0,2 \pi)$, and define, for any $u \in \widetilde{\Gamma}$, the path $v_{u}:[-1,2] \rightarrow \mathbb{R}^{2} \backslash \Sigma$ as

$$
v_{u}(t)=\left\{\begin{array}{ll}
u(t) & t \in[-1,1] \\
\left|q^{-}\right| e^{i\left(\theta^{+}+\left(\theta^{-}-\theta^{+}+2 \pi\right)(t-1)\right)} & t \in[1,2]
\end{array} \quad \text { if } \theta^{-}<\theta^{+},\right.
$$

and

$$
v_{u}(t)=\left\{\begin{array}{ll}
u(t) & t \in[-1,1] \\
\left|q^{-}\right| e^{i\left(\theta^{+}+\left(\theta^{-}-\theta^{+}\right)(t-1)\right)} & t \in[1,2]
\end{array} \quad \text { if } \theta^{+}<\theta^{-},\right.
$$

namely we artificially close the path $u$ with the arc on $\partial B_{\left|q^{-}\right|}$connecting $q^{+}$with $q^{-}$in the counterclockwise sense. With this notation, and given $l \in \mathbb{Z}_{2}^{N}$, we introduce the set

$$
\widehat{\Gamma}_{l}=\left\{u \in \widehat{\Gamma}: \operatorname{Ind}\left(v_{u}, c_{i}\right) \equiv l_{i} \bmod 2, \forall i=1, \ldots, N\right\},
$$

being (in complex notation)

$$
\operatorname{Ind}\left(v_{u}, c_{i}\right)=\frac{1}{2 \pi i} \int_{v_{u}} \frac{\mathrm{d} z}{z-c_{i}}
$$

the usual winding number of a closed planar path. We are now in position to prove the following result:

Theorem 2.1 Let $q^{-}, q^{+}$be as in (8) and let $l \in \mathbb{Z}_{2}^{N}$ satisfying

$$
\exists k \neq m: l_{k} \neq l_{m} .
$$

Then, there exists a self-intersection-free parabolic solution of (6), corresponding to a (collision-free) minimizer of $\mathcal{M}$ in the $H^{1}$-weak closure of $\widehat{\Gamma}_{l}$.

Proof The existence of a minimizer $u$ of $\mathcal{M}$ in the $H^{1}$-weak closure of $\widehat{\Gamma}_{l}$ follows from standard lower-semicontinuity/coercivity arguments; notice, however, that the coercivity of $\mathcal{M}$ is not straightforward, following from the assumption at infinity (5) (see [9, Lemma 4.2] for the details). The fact that $u$ is collision-free can be proved as in [18, Theorem 4.12] or in [19, Theorem 2.3], using (4) in an essential way and taking into account that the assumption 
$q^{-} \neq q^{+}$rules out the case of collision-ejection solutions. Finally, the fact that $u$ is selfintersection free follows as in [18, Theorem 4.12] again (see, in particular, [18, Proposition 4.24]).

\section{Entire parabolic solutions}

In this section we prove Theorem 1.1 via an approximation argument. More precisely, given $\xi^{-}, \xi^{+} \in \mathbb{S}^{1}$ with $\xi^{-} \neq \xi^{+}$and a partition $\mathcal{P}$ of $\Sigma$, we first define $l \in \mathbb{Z}_{2}^{N}$ by setting $l_{i}=1$ if and only if $c_{i} \in \mathcal{P}$ and we apply Theorem 2.1 with the choice $q^{ \pm}=R \xi^{ \pm}$for $R>0$ large enough (notice that in this way (8) is surely satisfied) so as to find an associated parabolic solution $x_{R}:\left[-\omega_{R}, \omega_{R}\right] \rightarrow \mathbb{R}^{2}$; then, we are going to show that an entire parabolic solution $x: \mathbb{R} \rightarrow \mathbb{R}^{2}$ can be obtained by passing to the limit when $R \rightarrow+\infty$.

In order to do this, the assumption at infinity (5) will play a crucial role. For further convenience, we fix from the beginning two constants $C_{-}, C_{+}>0$ and a constant $K>$ $\sup _{i}\left|c_{i}\right|+1$ such that

$$
\begin{gathered}
|W(x)| \leq \frac{C_{+}}{|x|^{\beta}} \quad \text { and } \quad|\nabla W(x)| \leq \frac{C_{+}}{|x|^{\beta+1}}, \quad \text { for every }|x| \geq K, \\
2|W(x)|+|\nabla W(x) \cdot x| \leq \frac{(2-\alpha) m}{2 \alpha} \frac{1}{|x|^{\alpha}}, \quad \text { for every }|x| \geq K, \\
\quad \frac{C_{-}}{|x|^{\alpha}} \leq U(x) \leq \frac{C_{+}}{|x|^{\alpha}}, \quad \text { for every }|x| \geq K,
\end{gathered}
$$

and

$$
\sqrt{\frac{m}{\alpha}} \frac{1}{|x|^{\alpha / 2}}-\frac{C_{+}}{|x|^{\beta-\alpha / 2}} \leq \sqrt{U(x)} \leq \sqrt{\frac{m}{\alpha}} \frac{1}{|x|^{\alpha / 2}}+\frac{C_{+}}{|x|^{\beta-\alpha / 2}}, \quad \text { for every }|x| \geq K .
$$

The estimates (11), (12) and (13) are rather obvious, while (14) follows from (11) using the elementary inequalities $1-|s| \leq \sqrt{1+s} \leq 1+\frac{1}{2} s$ (valid for $s \geq-1$ ).

We are now in position to give the proof; as a useful notation, we set $r_{R}(t)=\left|x_{R}(t)\right|$ and, whenever $r_{R}(t) \neq 0, s_{R}(t)=\frac{x_{R}(t)}{r_{R}(t)}$. We split our arguments into several steps; first of all, we observe that due to the assumption (10) any solution $x_{R}$ enters the ball $B_{K}$, so that

$$
\limsup _{R \rightarrow+\infty} \min _{t} r_{R}(t) \leq K<+\infty \text {. }
$$

\subsection{The virial identity and some preliminary estimates}

Preliminary, we observe that, due to the fact that $x_{R}$ has zero-energy (see 7), the following equality—often referred to as virial identity—holds true:

$$
\frac{\mathrm{d}^{2}}{\mathrm{~d} t^{2}}\left(\frac{1}{2} r_{R}(t)^{2}\right)=2 U\left(x_{R}(t)\right)+\nabla U\left(x_{R}(t)\right) \cdot x_{R}(t) .
$$

Using (5) and (12), we see that the above expression is strictly positive for $\left|x_{R}(t)\right| \geq K$, precisely

$$
\frac{\mathrm{d}^{2}}{\mathrm{~d} t^{2}}\left(\frac{1}{2} r_{R}(t)^{2}\right) \geq \frac{(2-\alpha) m}{2 \alpha r_{R}(t)^{\alpha}} .
$$

Therefore, $t_{0} \in\left(-\omega_{R}, \omega_{R}\right)$ can be a local maximum for $t \mapsto r_{R}(t)$ only if $r_{R}\left(t_{0}\right)<K$. 
On the one hand, this implies that $r_{R}(t)<R$ for every $t \in\left(-\omega_{R}, \omega_{R}\right)$. As a consequence, $x_{R}$ separates the set $\Sigma$ according the partition $\mathcal{P}$, in the sense specified in [18, pp. 3263-3264] (that is to say, when closing the path $x_{R}$ as described in Sect. 2 so as to find a Jordan curve $\gamma_{R}$, two centres lie in the same connected component of $\mathbb{R}^{2} \backslash \gamma_{R}$ if and only if they are both in $\mathcal{P}$ or both in $\Sigma \backslash \mathcal{P}$ ).

On the other hand, it follows that there are exactly two instants $t_{R}^{ \pm} \in\left(-\omega_{R}, \omega_{R}\right)$, with $t_{R}^{-}<t_{R}^{+}$, such that $r_{R}\left(t_{R}^{ \pm}\right)=K$ (implying $r_{R}(t)<K$ for $t \in\left(t_{R}^{-}, t_{R}^{+}\right)$and $r_{R}(t)>K$ for $\left.t \notin\left[t_{R}^{-}, t_{R}^{+}\right]\right)$; moreover, $\dot{r}_{R}(t) \neq 0$ for $t \notin\left(t_{R}^{-}, t_{R}^{+}\right)$. Using the fact that $x_{R}$ has zero-energy together with (13), we also find

$$
\begin{aligned}
\omega_{R}-t_{R}^{+} & =\int_{t_{R}^{+}}^{\omega_{R}} \frac{\dot{r}_{R}(t)}{\dot{r}_{R}(t)} \mathrm{d} t \geq \frac{1}{\sqrt{2 C_{+}}} \int_{t_{R}^{+}}^{\omega_{R}} \frac{\dot{r}_{R}(t)}{r_{R}(t)^{-\alpha / 2}} \mathrm{~d} t \\
& =\frac{1}{(1+\alpha / 2) \sqrt{2 C_{+}}}\left(R^{1+\alpha / 2}-K^{1+\alpha / 2}\right),
\end{aligned}
$$

implying that $\omega_{R}-t_{R}^{+} \rightarrow+\infty$ for $R \rightarrow+\infty$. Analogously, $-\omega_{R}-t_{R}^{-} \rightarrow-\infty$.

For the rest of the proof, it is convenient to suppose $t_{R}^{-}=-t_{R}^{+}$, that is, the time spent by $x_{R}$ inside the ball $B_{K}$ is a symmetric interval with respect to the origin. This is not restrictive, up to a ( $R$-dependent) time shift of the solution $x_{R}$. With a slight abuse of notation, we will still denote by $x_{R}$ this time translation, and by $\left[\omega_{R}^{-}, \omega_{R}^{+}\right]$its interval of definition.

\subsection{Passing to the limit: a generalized solution}

In this step, we show how to pass to the limit when $R \rightarrow+\infty$, so as to find an entire generalized solution, that is, a solution with a zero-measure (but possibly non-empty) set of collision instants, see [2]. For the next arguments, we write

$$
\mathcal{A}_{[a, b]}(x)=\int_{a}^{b}\left(\frac{1}{2}|\dot{x}(t)|^{2}+U(x(t))\right) \mathrm{d} t
$$

for the action of an $H^{1}$-path $x:[a, b] \rightarrow \mathbb{R}^{2} \backslash \Sigma$; notice that, whenever $x$ satisfies the zero-energy relation (7), we have

$$
\mathcal{A}_{[a, b]}(x)=\int_{a}^{b}|\dot{x}(t)|^{2} \mathrm{~d} t=2 \int_{a}^{b} U(x(t)) \mathrm{d} t=\sqrt{2} \int_{a}^{b}|\dot{x}(t)| \sqrt{U(x(t))} \mathrm{d} t .
$$

Having introduced this notation, the crucial point will be to prove that

$$
\limsup _{R \rightarrow+\infty} \mathcal{A}_{\left[t_{R}^{-}, t_{R}^{+}\right]}\left(x_{R}\right)<+\infty
$$

with $t_{R}^{ \pm}$defined by the previous step. From this, several facts can be deduced. Precisely, since

$$
\left(\inf _{|x| \leq K} U(x)\right)\left(t_{R}^{+}-t_{R}^{-}\right) \leq \int_{t_{R}^{-}}^{t_{R}^{+}} U\left(x_{R}(t)\right) \mathrm{d} t,
$$

we get at first that $t_{R}^{+}-t_{R}^{-}$is bounded, say $t_{R}^{+}-t_{R}^{-} \leq 2 T$ for any $R$. From this, together with the fact that $\left|x_{R}(t)\right| \leq K$ for $t \in\left[t_{R}^{-}, t_{R}^{+}\right]$and with (16) again, we infer that

$$
\left\|x_{R}\right\|_{H^{1}\left(t_{R}^{-}, t_{R}^{+}\right)}^{2}=\int_{t_{R}^{-}}^{t_{R}^{+}}\left(\left|x_{R}(t)\right|^{2}+\left|\dot{x}_{R}(t)\right|^{2}\right) \mathrm{d} t
$$


is bounded as well. Using, moreover, the fact that $\left|\ddot{x}_{R}(t)\right| \leq\left(\sup _{|x| \geq K} U(x)\right)$ for $t \notin\left[t_{R}^{-}, t_{R}^{+}\right]$, together with the boundedness of $\left|x_{R}\left(t_{R}^{ \pm}\right)\right|$and of $\left|\dot{x}_{R}\left(t_{R}^{ \pm}\right)\right|=\sqrt{2 U\left(x_{R}\left(t_{R}^{ \pm}\right)\right)}$, we finally conclude that $x_{R}$ is bounded in $H_{\text {loc }}^{1}(\mathbb{R})$. As a consequence, there exists an $H^{1}$-function $x_{\infty}: \mathbb{R} \rightarrow \mathbb{R}^{2}$ such that $x_{R} \rightarrow x_{\infty}$ weakly in $H_{\text {loc }}^{1}(\mathbb{R})$ (in particular, uniformly on compact sets) for $R \rightarrow+\infty$. Of course, $x_{\infty}$ turns out to be a parabolic solution of (2) as long as it does not collide with the set of the centres; moreover, $\left|x_{\infty}(t)\right| \geq K$ for $|t| \geq T$ so that the arguments of Sect. 3.1 imply that $x_{\infty}$ is unbounded for $t \rightarrow \pm \infty$. Finally, by the $H_{\text {loc }}^{1}$-boundedness and Fatou's lemma,

$$
\int_{-T}^{T} U\left(x_{\infty}(t)\right) \mathrm{d} t \leq \liminf _{R \rightarrow+\infty} \int_{-T}^{T} U\left(x_{R}(t)\right) \mathrm{d} t=\liminf _{R \rightarrow+\infty} \frac{1}{2} \int_{-T}^{T}\left|\dot{x}_{R}(t)\right|^{2} \mathrm{~d} t<\infty,
$$

implying that the set of collision instants has zero measure.

The rest of this subsection is then devoted to the proof of (16). We are going to show that

$$
\mathcal{A}_{\left[\omega_{R}^{-}, \omega_{R}^{+}\right]}\left(x_{R}\right) \leq\left(\sqrt{\frac{2 m}{\alpha}} \frac{4}{2-\alpha}\right) R^{1-\alpha / 2}+M
$$

and that

$$
\mathcal{A}_{\left[\omega_{R}^{-}, t_{R}^{-}\right] \cup\left[t_{R}^{+}, \omega_{R}^{+}\right]}\left(x_{R}\right) \geq\left(\sqrt{\frac{2 m}{\alpha}} \frac{4}{2-\alpha}\right) R^{1-\alpha / 2}-M,
$$

for some constant $M>0$, from which (16) clearly follows.

We first prove (17). To this end, let us define the $H^{1}$-path

$$
\zeta(t)= \begin{cases}\xi^{+} \eta^{+}(t) & \text { for } t \in\left[1, \Theta_{R}^{+}\right] \\ \gamma(t) & \text { for } t \in[-1,1] \\ \xi^{-} \eta^{-}(t) & \text { for } t \in\left[\Theta_{R}^{-},-1\right]\end{cases}
$$

where $\gamma$ is an arbitrary $H^{1}$-path joining the points $K \xi^{-}$and $K \xi^{+}$and separating the set according to the partition $\mathcal{P}$ (in the sense specified in Sect. 2), $\eta^{+}:[1,+\infty) \rightarrow[K,+\infty)$ and $\eta^{-}:(-\infty,-1] \rightarrow[K,+\infty)$ are the solutions of the Cauchy problems

$$
\dot{\eta}^{ \pm}= \pm \sqrt{2 U\left(\xi^{ \pm} \eta^{ \pm}\right)}, \quad \eta^{ \pm}( \pm 1)=K
$$

and $\Theta_{R}^{+}, \Theta_{R}^{-}$(for $R>K$ ) are the unique points such that $\eta^{ \pm}\left(\Theta_{R}^{ \pm}\right)=R$. Then, we set

$$
\tilde{\zeta}(t)=\zeta\left(\Theta_{R}^{-}+\frac{1}{2}\left(\Theta_{R}^{+}-\Theta_{R}^{-}\right)(t+1)\right), \quad \text { for any } t \in[-1,1],
$$

in such a way that $\tilde{\zeta}$ is an $H^{1}$-path defined on $[-1,1]$, joining the points $R \xi^{-}$and $R \xi^{+}$and separating the set according to the partition $\mathcal{P}$. Using the well-known relation

$$
\frac{1}{\sqrt{2}} \mathcal{A}_{\left[\omega_{R}^{-}, \omega_{R}^{+}\right]}\left(x_{R}\right)=\sqrt{\mathcal{M}\left(u_{R}\right)}, \quad \text { with } u_{R}(t)=x_{R}\left(\omega_{R}^{-}+\frac{1}{2}\left(\omega_{R}^{+}-\omega_{R}^{-}\right)(t+1)\right),
$$

together with the minimality of $u_{R}$ in the corresponding homotopy class, we find

$$
\frac{1}{\sqrt{2}} \mathcal{A}_{\left[\omega_{R}^{-}, \omega_{R}^{+}\right]}\left(x_{R}\right) \leq \sqrt{\mathcal{M}(\tilde{\zeta})} .
$$


We, therefore, compute

$$
\begin{aligned}
\sqrt{\mathcal{M}(\tilde{\zeta})}= & \frac{1}{\sqrt{2}} \inf _{\Theta>0} \mathcal{A}_{[-\Theta, \Theta]}(\tilde{\zeta}(\cdot / \Theta)) \leq \frac{1}{\sqrt{2}} \int_{\Theta_{R}^{-}}^{\Theta_{R}^{+}}\left(\frac{1}{2}|\dot{\zeta}(t)|^{2}+U(\zeta(t))\right) \mathrm{d} t \\
& \leq \frac{1}{\sqrt{2}} \int_{\Theta_{R}^{-}}^{-1}\left(\frac{1}{2}\left|\dot{\eta}^{-}(t)\right|^{2}+U\left(\xi^{-} \eta^{-}(t)\right)\right) \mathrm{d} t+\frac{1}{\sqrt{2}} \mathcal{A}_{[-1,1]}(\gamma) \\
& +\frac{1}{\sqrt{2}} \int_{1}^{\Theta_{R}^{+}}\left(\frac{1}{2}\left|\dot{\eta}^{+}(t)\right|^{2}+U\left(\xi^{+} \eta^{+}(t)\right)\right) \mathrm{d} t \\
= & M_{+}+\int_{-1}^{\Theta_{R}^{-}} \sqrt{U\left(\xi^{-} \eta^{-}(t)\right)} \dot{\eta}^{-}(t) \mathrm{d} t+\int_{1}^{\Theta_{R}^{+}} \sqrt{U\left(\xi^{+} \eta^{+}(t)\right)} \dot{\eta}^{+}(t) \mathrm{d} t \\
= & M_{+}+\int_{K}^{R} \sqrt{U\left(\xi^{-} r\right)} \mathrm{d} r+\int_{K}^{R} \sqrt{U\left(\xi^{+} r\right)} \mathrm{d} r
\end{aligned}
$$

with $M_{+}=\frac{1}{\sqrt{2}} \mathcal{A}_{[-1,1]}(\gamma)$ (not depending on $R$ ). Now, using the estimate from above in (14) we find

$$
\sqrt{U\left(\xi^{ \pm} r\right)} \leq \sqrt{\frac{m}{\alpha}} \frac{1}{r^{\alpha / 2}}+\frac{C_{+}}{r^{\beta-\alpha / 2}}, \quad \text { for every } r \geq K,
$$

so that, with a simple computation,

$$
\sqrt{\mathcal{M}(\tilde{\zeta})} \leq\left(\sqrt{\frac{m}{\alpha}} \frac{4}{2-\alpha}\right) R^{1-\alpha / 2}+M_{+}+\frac{4 C_{+}}{2 \beta-\alpha-2},
$$

finally implying (17). To prove (18), we write

$$
\mathcal{A}_{\left[\omega_{R}^{-}, t_{R}^{-}\right] \cup\left[t_{R}^{+}, \omega_{R}^{-}\right]}\left(x_{R}\right)=\sqrt{2} \int_{\left[\omega_{R}^{-}, t_{R}^{-}\right] \cup\left[t_{R}^{+}, \omega_{R}^{-}\right]}\left|\dot{x}_{R}(t)\right| \sqrt{U\left(x_{R}(t)\right)} \mathrm{d} t
$$

and we observe that $\left|\dot{x}_{R}(t)\right| \geq\left|\dot{r}_{R}(t)\right|$; moreover, by the arguments in Sect. 3.1, $\dot{r}_{R}(t)<0$ for $t \in\left[\omega_{R}^{-}, t_{R}^{-}\right]$and $\dot{r}_{R}(t)>0$ for $t \in\left[t_{R}^{+}, \omega_{R}^{+}\right]$. Hence, using the estimate from below (14) yields the conclusion.

\subsection{Asymptotic directions}

We now prove that the (generalized) solution $x_{\infty}$ has $\xi^{ \pm}$has asymptotic directions for $t \rightarrow$ $\pm \infty$, respectively; more precisely, writing $s_{\infty}(t)=\frac{x_{\infty}(t)}{\left|x_{\infty}(t)\right|}$ for $|t| \geq T$, we are going to show that $s_{\infty}( \pm \infty)=\xi^{ \pm}$. Throughout this step of the proof, we assume that the solution $x_{R}$ is defined on the whole real line, as well. This is not restrictive, since the arguments of Sect. 3.1 (together with the boundedness of $\nabla U$ at infinity) rule out the occurrence of blowup phenomena, and of course does not have influence on the local convergence $x_{R} \rightarrow x_{\infty}$; however, it turns out to be useful since it allows to perform estimates valid for any $t$ large enough (in absolute value).

We give the details for $t \rightarrow+\infty$. As a first step, we prove that

$$
r_{R}(t) \geq\left(\frac{(2-\alpha) m}{2 \alpha}\right)^{\frac{1}{\alpha+2}}\left(t-t_{R}^{+}\right)^{\frac{2}{\alpha+2}}, \quad \text { for every } t \geq t_{R}^{+} .
$$


To obtain the above inequality, we first integrate (15) on $\left[t_{R}^{+}, s\right]$, recalling that $r_{R}(s) \leq r_{R}(t)$ whenever $t_{R}^{+} \leq s \leq t$, so as to obtain

$$
\frac{\mathrm{d}}{\mathrm{d} t}\left(\frac{1}{2} r_{R}(s)^{2}\right) \geq \frac{(2-\alpha) m}{2 \alpha m} \frac{\left(s-t_{R}^{+}\right)}{r_{R}(t)^{\alpha}}, \quad \text { for every } t \geq s ;
$$

a further integration on $\left[t_{R}^{+}, t\right]$ thus yields (19).

Taking into account that $t_{R}^{+} \leq T$, it follows from (19) that there exists $\widehat{T}>T$ such that $r_{R}(t) \geq K+1$ for $t \geq \widehat{T}$. We now claim that

$$
\left|\dot{s}_{R}(t)\right| \leq \frac{C}{(t-T)^{\frac{4}{\alpha+2}}}, \quad \text { for every } t \geq \widehat{T},
$$

where $C>0$ is a suitable constant depending only on the potential (and on $K$ ). To prove this, we define $A_{R}(t)=x_{R}(t) \wedge \dot{x}_{R}(t)$. Taking into account (5) and (11), we first obtain from (19) that

$$
\left|\dot{A}_{R}(t)\right|=\left|x_{R}(t) \wedge \nabla W\left(x_{R}(t)\right)\right| \leq \frac{C_{+}}{r_{R}^{\beta}(t)} \leq C_{+}\left[\frac{2 \alpha}{(2-\alpha) m}\right]^{\beta /(\alpha+2)} \frac{1}{\left(t-t_{R}^{+}\right)^{2 \beta /(\alpha+2)}}
$$

for every $t \geq t_{R}^{+}$. Denoting by $\hat{t}_{R} \in\left(t_{R}^{+}, \widehat{T}\right]$ the (unique) instant such that $r_{R}\left(\hat{t}_{R}\right)=K+1$, we then obtain, for $t \geq \widehat{T}$,

$$
\begin{aligned}
\left|A_{R}(t)\right| & \leq\left|x_{R}\left(\hat{t}_{R}\right)\right|\left|\dot{x}_{R}\left(\hat{t}_{R}\right)\right|+\int_{\hat{t}_{R}}^{+\infty}\left|\dot{A}_{R}(\tau)\right| \mathrm{d} \tau \\
& \leq r_{R}\left(\hat{t}_{R}\right) \sqrt{2 U\left(x_{R}\left(\hat{t}_{R}\right)\right)}+\int_{\hat{t}_{R}}^{+\infty}\left|\dot{A}_{R}(\tau)\right| \mathrm{d} \tau,
\end{aligned}
$$

where

$$
\begin{aligned}
r_{R}\left(\hat{t}_{R}\right) \sqrt{2 U\left(x_{R}\left(\hat{t}_{R}\right)\right)} & \leq \sqrt{2 C_{+}}(K+1)^{\frac{2-\alpha}{2}} \\
\int_{\hat{t}_{R}}^{+\infty}\left|\dot{A}_{R}(\tau)\right| d \tau & \leq C_{+}\left[\frac{2 \alpha}{(2-\alpha) m}\right]^{\beta /(\alpha+2)} \frac{\alpha+2}{2 \beta-\alpha-2} \frac{1}{\left(\hat{t}_{R}-t_{R}^{+}\right)^{(2 \beta-\alpha-2) /(\alpha+2)}} \\
\hat{t}_{R}-t_{R}^{+} & \geq \frac{1}{(1+\alpha / 2) \sqrt{2 C_{+}}}\left[(K+1)^{1+\alpha / 2}-K^{1+\alpha / 2}\right]
\end{aligned}
$$

using (13) to bound from above $U\left(x_{R}\left(\hat{t}_{R}\right)\right)$. We have argued as in Sect. 3.1 to bound from below the quantity $\hat{t}_{R}-t_{R}^{+}$. Observing that $\left|\dot{s}_{R}(t)\right|=\frac{\left|A_{R}(t)\right|}{\left|r_{R}(t)\right|^{2}}$ and using (19) once again, (20) finally follows.

From this we can easily conclude. Indeed, on the one hand, Lebesgue's theorem is seen to apply, giving (together with uniform convergence on compact sets),

$$
s_{R}(+\infty)=s_{R}(\widehat{T})+\int_{\widehat{T}}^{\infty} \dot{s}_{R}(\tau) \mathrm{d} \tau \rightarrow s_{\infty}(\widehat{T})+\int_{\widehat{T}}^{\infty} \dot{s}_{\infty}(\tau) \mathrm{d} \tau=s_{\infty}(+\infty)
$$

for $R \rightarrow+\infty$. On the other hand, recalling that $s_{R}\left(\omega_{R}^{+}\right)=\xi^{+}$and using (20) again,

$$
s_{R}(+\infty)=\xi^{+}+\int_{\omega_{R}^{+}}^{+\infty} \dot{s}_{R}(\tau) \mathrm{d} \tau \rightarrow \xi^{+},
$$

finally yielding $s_{\infty}(+\infty)=\xi^{+}$. The proof that $s_{\infty}(-\infty)=\xi^{-}$is analogous. 


\subsection{Avoiding collisions}

In this step, we rule out the occurrence of collisions for $x_{\infty}$, that is, we prove that $x_{\infty}(t) \notin \Sigma$ for any $t \in \mathbb{R}$. We need to distinguish two cases, depending on whether $\alpha \in(1,2)$ or $\alpha=1$.

Let us suppose that $\alpha \in(1,2)$. Assume by contradiction that $x_{\infty}^{-1}(\Sigma) \neq \emptyset$; to fix the ideas, suppose that $x_{\infty}$ has (at least one) collision with the centre $c_{1}$ and take $\delta^{*}>0$ so small that $c_{i} \notin B_{\delta^{*}}\left(c_{1}\right)$ for $i=2, \ldots, N$. Then, it is possible to find $\tau_{R}^{-}, \tau_{R}, \tau_{R}^{+} \in\left(t_{R}^{-}, t_{R}^{+}\right)$such that $\tau_{R}^{-}<\tau_{R}<\tau_{R}^{+}, \delta_{R}:=\left|x_{R}\left(\tau_{R}\right)-c_{1}\right|=\min _{t}\left|x_{R}(t)-c_{1}\right| \rightarrow 0^{+}$,

$$
\left|x_{R}\left(\tau_{R}^{ \pm}\right)-c_{1}\right|=\delta^{*} \quad \text { and } \quad\left|x_{R}(t)-c_{1}\right| \leq \delta^{*}, \quad \text { for any } t \in\left[\tau_{R}^{-}, \tau_{R}^{+}\right] .
$$

Since $t_{R}^{+}-t_{R}^{-}$is bounded and $x_{R} \rightarrow x_{\infty}$ uniformly on compact sets, both $\tau_{R}-\tau_{R}^{-}$and $\tau_{R}^{+}-\tau_{R}$ are bounded away from zero. Let us define

$$
v_{R}(t)=\frac{1}{\delta_{R}}\left(x_{R}\left(\delta_{R}^{1+\alpha / 2} t+\tau_{R}\right)-c_{1}\right), \quad t \in\left[-\gamma_{R}, \sigma_{R}\right],
$$

where

$$
-\gamma_{R}=\frac{\tau_{R}^{-}-\tau_{R}}{\delta_{R}^{1+\alpha / 2}} \text { and } \sigma_{R}=\frac{\tau_{R}^{+}-\tau_{R}}{\delta_{R}^{1+\alpha / 2}} .
$$

Notice that $-\gamma_{R} \rightarrow-\infty$ and $\sigma_{R} \rightarrow+\infty,\left|v_{R}(0)\right|=1,\left|v_{R}(t)\right| \geq 1$ and $\left|\delta_{R} v_{R}(t)\right| \leq \delta^{*}$ for $t \in\left[-\gamma_{R}, \sigma_{R}\right]$. An easy computation shows that, writing $U$ as in (4), $v_{R}$ satisfies

$$
\ddot{v}_{R}=-\frac{m_{1} v_{R}}{\left|v_{R}\right|^{\alpha+2}}+\delta_{R}^{1+\alpha} \nabla U_{1}\left(\delta_{R} v_{R}+c_{1}\right)
$$

and

$$
\frac{1}{2}\left|\dot{v}_{R}\right|^{2}=\frac{m_{1}}{\alpha\left|v_{R}\right|^{\alpha}}+\delta_{R}^{\alpha} U_{1}\left(\delta_{R} v_{R}+c_{1}\right) .
$$

As a consequence, it is easy to see that $v_{R} \rightarrow v_{\infty}$ in $\mathcal{C}_{\text {loc }}^{2}(\mathbb{R})$, where $v_{\infty}$ is a zero-energy solution of

$$
\ddot{v}_{\infty}=-\frac{m_{1} v_{\infty}}{\left|v_{\infty}\right|^{\alpha+2}}
$$

By [9, Proposition 6.1], $v_{\infty}$ has transversal self-intersections. Since transversal selfintersections are stable with respect to small perturbations, this contradicts the fact that $x_{R}$ (and hence $v_{R}$ ) is self-intersection free, thus ending the proof.

Assume instead that $\alpha=1$. Keeping the previous notation (and assuming now, up to passing to a subsequence, the existence of the limit $\tau_{R} \rightarrow \tau_{\infty}$ ) we define the Sundman integral

$$
s_{R}(t)=\int_{\tau_{R}}^{t} \frac{\mathrm{d} \tau}{\left|x_{R}(\tau)-c_{1}\right|}, \quad t \in\left[\tau_{R}^{-}, \tau_{R}^{+}\right],
$$

and we use (with the usual identification $\mathbb{R}^{2} \cong \mathbb{C}$ ) the well-known Levi-Civita change of variables

$$
w_{R}(s)^{2}=x_{R}\left(t_{R}(s)\right)-c_{1}, \quad s \in\left[\sigma_{R}^{-}, \sigma_{R}^{+}\right],
$$

being $t_{R}$ the inverse of $s_{R}$ and $\sigma_{R}^{ \pm}=s_{R}\left(\tau_{R}^{ \pm}\right)$. Notice that the above change of variables is not one-to-one; however, we can uniquely define $w_{R}$ by writing in polar coordinates $x_{R}-c_{1}=\rho_{R} e^{i \varphi_{R}}$ and setting $w_{R}=\rho_{R}^{1 / 2} e^{i \varphi / 2}$. Also, observe that both $\sigma_{R}^{-}$and $\sigma_{R}^{+}$are bounded away from zero, since $\left|s_{R}(t)\right| \geq\left|t-\tau_{R}\right| / \delta^{*}$. 
Standard computations yield:

$$
\ddot{x}_{R}=2 w_{R}^{\prime \prime} \frac{w_{R}}{\left|w_{R}\right|^{4}}=-2 \frac{w_{R}^{2}\left|w_{R}^{\prime}\right|^{2}}{\left|w_{R}\right|^{6}} .
$$

Here and in what follows all functions $w_{R}$ and their derivatives are evaluated at $s=s_{R}(t)$. Using the equation and writing $U$ as in (4) with $\alpha=1$ we get

$$
2 w_{R}^{\prime \prime} w_{R}=2 \frac{w_{R}^{2}\left|w_{R}^{\prime}\right|^{2}}{\left|w_{R}\right|^{2}}-\frac{m_{1} w_{R}^{2}}{\left|w_{R}\right|^{2}}+\left|w_{R}\right|^{4} \nabla U_{1}\left(c_{1}+w_{R}^{2}\right)
$$

which gives

$$
w_{R}^{\prime \prime}=\frac{w_{R}}{\left|w_{R}\right|}\left|w_{R}^{\prime}\right|^{2}-\frac{m_{1} w_{R}}{2\left|w_{R}\right|^{2}}+\frac{\bar{w}_{R}}{2}\left|w_{R}\right|^{2} \nabla U_{1}\left(c_{1}+w_{R}^{2}\right)
$$

once it is multiplied by the complex conjugate $\bar{w}_{R}$. Finally, the zero-energy relation for $x_{R}$ yields

$$
w_{R}^{\prime \prime}=\frac{w_{R}}{2} U_{1}\left(c_{1}+w_{R}^{2}\right)+\frac{\bar{w}_{R}}{2}\left|w_{R}\right|^{2} \nabla U_{1}\left(c_{1}+w_{R}^{2}\right)
$$

moreover

$$
\left|w_{R}(0)\right|=\left|x_{R}\left(\tau_{R}\right)-c_{1}\right|=\delta_{R} \rightarrow 0
$$

and

$$
\left|w_{R}^{\prime}(0)\right|^{2}=\frac{m_{1}}{2}+\frac{\left|w_{R}(0)\right|^{2}}{2} U_{1}\left(c_{1}+w_{R}(0)^{2}\right) \rightarrow \frac{m_{1}}{2} .
$$

By a continuous dependence argument, $w_{R}$ converges (up to subsequences) uniformly on compact intervals containing the origin to the solution $w_{\infty}$ of the Cauchy problem associated with (21) having initial conditions $w_{\infty}(0)=0$ and $w_{\infty}(0)=v$ for some $|v|^{2}=\frac{m_{1}}{2}$; moreover, the symmetries of differential Eq. (21) imply that it must be $w_{\infty}(-s)=-w_{\infty}(s)$ for any $s$ small enough.

It follows that

$$
t_{R}(s)=\tau_{R}+\int_{0}^{s}\left|w_{R}(\sigma)\right|^{2} \mathrm{~d} \sigma \rightarrow t_{\infty}(s)=\tau_{\infty}+\int_{0}^{s}\left|w_{\infty}(\sigma)\right|^{2} \mathrm{~d} \sigma
$$

uniformly on compact sets for $R \rightarrow+\infty$; moreover, the map $s \mapsto t_{\infty}(s)-\tau^{*}$ is an odd function. Taking into account that $x_{R} \rightarrow x_{\infty}$ uniformly on compact sets, we find

$$
w_{\infty}(s)^{2}=x_{\infty}\left(t_{\infty}(s)\right)-c_{1}, \quad \text { for every } s \text { small enough, }
$$

finally implying that $x_{\infty}(t) \neq c_{1}$ for $t$ near $\tau_{\infty}$ and that

$$
x_{\infty}\left(\tau_{\infty}-t\right)=x_{\infty}\left(\tau_{\infty}+t\right), \quad \text { for every } t \text { small enough. }
$$

Since $x_{\infty}$ is a classical solution of (2) outside the collision set, and possibly repeating the above argument for any collision instant, we find a contradiction with the global property that $x_{\infty}$ has different asymptotic directions for $t \rightarrow \pm \infty$. 


\subsection{Conclusion}

To conclude, we only need to show that $x_{\infty}$ is self-intersection free and that has the desired topological characterization. Actually, this second property immediately follows from the first one (taking into account the topological characterization of $x_{R}$ ), so let us show that $x_{\infty}$ is self-intersection free. Of course, transversal self-intersections are ruled out since $x_{R}$ is self-intersection free. On the other hand, assume by contradiction that there is a tangential self-intersection, that is, $x_{\infty}\left(t_{1}\right)=x_{\infty}\left(t_{2}\right)$ and $\dot{x}_{\infty}\left(t_{1}\right)$ parallel to $\dot{x}_{\infty}\left(t_{2}\right)$ for some $t_{1} \neq t_{2}$. Then, the zero-energy condition gives $\left|\dot{x}_{\infty}\left(t_{1}\right)\right|=\left|\dot{x}_{\infty}\left(t_{2}\right)\right|$, so that $\dot{x}_{\infty}\left(t_{1}\right)= \pm \dot{x}_{\infty}\left(t_{2}\right)$. Both the cases are not possible in view of the local uniqueness to the Cauchy problems. More precisely, in the first one $x_{\infty}$ should be periodic, while in the second one it should be $x_{\infty}(t)=x_{\infty}\left(t_{2}+t_{1}-t\right)$, which implies that $\dot{x}_{\infty}\left(\left(t_{2}+t_{1}\right) / 2\right)=0$ contradicting the conservation of energy.

Remark 3.1 If $\alpha \in(1,2)$ then the conclusion of Theorem 1.1 still holds true when $\xi^{+}=\xi^{-}$. In fact we observe that, if $\alpha>1$, then Theorem 2.1 holds also for $q^{+}=q^{-}$(see [19]). Moreover, condition (10) grants that the minimizer $u$ of $\mathcal{M}$ in $\widehat{\Gamma}_{l}$ is not constant and that its value $\omega$ in (9) is positive. Therefore, it is possible to repeat the argument of Sect. 3 which does not use the assumption $\xi^{+} \neq \xi^{-}$for $\alpha \in(1,2)$.

Remark 3.2 Arguing as in [9, Proposition 2.4], it is possible to prove that the solution given by Theorem 1.1 satisfies the asymptotic estimate

$$
|x(t)| \sim\left(\sqrt{\frac{m}{2 \alpha}}(2+\alpha)\right)^{\frac{2}{2+\alpha}}|t|^{\frac{2}{2+\alpha}},
$$

when $t \rightarrow \pm \infty$.

Remark 3.3 We finally briefly describe the behaviour of the above found parabolic solutions when collapsing all the centres into a single one. In order to do this, we consider the parameterdependent $N$-centre problem

$$
\ddot{y}_{\varepsilon}=-\sum_{i=1}^{N} \frac{m_{i}\left(y-\varepsilon c_{i}\right)}{\left|y-\varepsilon c_{i}\right|^{\alpha+2}}
$$

when $\varepsilon \rightarrow 0^{+}$. Using a rescaling argument, solutions to the above equation can be obtained starting from solutions of (1). More precisely, if $x$ denotes an entire parabolic solution of (1), then the function

$$
y_{\varepsilon}(t)=\varepsilon x\left(\frac{t}{\varepsilon^{\frac{2+\alpha}{2}}}\right), \quad t \in \mathbb{R} ;
$$

is a zero-energy solution of (22). As a consequence of the asymptotic estimate given in Remark 3.2, we have that the pointwise limit of $y_{\varepsilon}(t)$ for $\varepsilon \rightarrow 0^{+}$exists, with

$$
\lim _{\varepsilon \rightarrow 0^{+}} y_{\varepsilon}(t)= \begin{cases}\left(\sqrt{\frac{m}{2 \alpha}}(2+\alpha)\right)^{\frac{2}{2+\alpha}}|t|^{\frac{2}{2+\alpha}} \xi^{-} & t<0, \\ 0 & t=0, \\ \left(\sqrt{\frac{m}{2 \alpha}}(2+\alpha)\right)^{\frac{2}{2+\alpha}}|t|^{\frac{2}{2+\alpha}} \xi^{+} & t>0 .\end{cases}
$$

As mentioned in the introduction, we have thus shown that, by collapsing all the centres into a single one, $y_{\varepsilon}$ converges to the juxtaposition of two rectilinear solutions of the $\alpha$-Kepler problem (actually, the convergence is easily seen to be $\mathcal{C}_{\text {loc }}^{2}(\mathbb{R} \backslash\{0\})$; compare with [22]). 
Acknowledgements We thank the two anonymous reviewers for their careful reading of the paper and the useful remarks and suggestions.

\section{References}

1. Ambrosetti, A., Coti Zelati, V.: Periodic Solutions of Singular Lagrangian Systems, Progress in Nonlinear Differential Equations and Their Applications, vol. 10. Birkhäuser Boston Inc, Boston (1993)

2. Bahri, A., Rabinowitz, P.H.: A minimax method for a class of Hamiltonian systems with singular potentials. J. Funct. Anal. 82, 412-428 (1989)

3. Barutello, V., Terracini, S., Verzini, G.: Entire parabolic trajectories as minimal phase transitions. Calc. Var. 49, 391-429 (2014)

4. Barutello, V., Terracini, S., Verzini, G.: Entire minimal parabolic trajectories: the planar anisotropic Kepler problem. Arch. Ration. Mech. Anal. 207, 583-609 (2013)

5. Bolotin, S.V.: Nonintegrability of the problem of $n$ centers for $n>2$, Vestnik Moskov. Univ. Ser. I Mat. Mekh. 1984(3), 65-68 (1984). (Russian)

6. Bolotin, S.V., Kozlov, V.V.: Topological approach to the generalized n-centre problem, (Russian). Uspekhi Mat. Nauk 72, 65-96 (2017). (English version on arXiv:1705.04671 [math.DS])

7. Bolotin, S.V., Negrini, P.: Chaotic behavior in the 3-center problem. J. Differ. Equ. 190, 539-558 (2003)

8. Bolotin, S.V., Negrini, P.: Regularization and topological entropy for the spatial $n$-center problem. Ergod. Theory Dyn. Syst. 21, 383-399 (2001)

9. Boscaggin, A., Dambrosio, W., Terracini, S.: Scattering parabolic solutions for the spatial $N$-centre problem. Arch. Ration. Mech. Anal. 223, 1269-1306 (2017)

10. Boscaggin, A., Garrione, M.: Positive solutions to indefinite Neumann problems when the weight has positive average. Discrete Contin. Dyn. Syst. 36, 5231-5244 (2016)

11. Castelli, R.: Topologically distinct collision-free periodic solutions for the $N$-center problem. Arch. Ration. Mech. Anal. 223, 941-975 (2017)

12. Chenciner, A.: Collisions totales, mouvements complètement paraboliques et réduction des homothéties dans le problème des $n$ corps (French). Regul. Chaotic Dyn. 3, 93-106 (1998)

13. Dimare, L.: Chaotic quasi-collision trajectories in the 3-centre problem. Celest. Mech. Dyn. Astron. 107, 427-449 (2010)

14. Klein, M., Knauf, A.: Classical planar scattering by Coulombic potentials. In: Lecture Notes in Physics, Springer, Berlin Heidelberg (1992)

15. Knauf, A.: The $n$-centre problem of celestial mechanics for large energies. J. Eur. Math. Soc. (JEMS) 4, $1-114$ (2002)

16. da Luz, A., Maderna, E.: On the free time minimizers of the Newtonian $N$-body problem. Math. Proc. Camb. Philos. Soc. 156, 209-227 (2014)

17. Maderna, E., Venturelli, A.: Globally minimizing parabolic motions in the Newtonian $N$-body problem. Arch. Ration. Mech. Anal. 194, 283-313 (2009)

18. Soave, N., Terracini, S.: Symbolic dynamics for the $N$-centre problem at negative energies. Discrete Contin. Dyn. Syst. 32, 3245-3301 (2012)

19. Soave, N., Terracini, S.: Avoiding collisions under topological constraints in variational problems coming from celestial mechanics. J. Fixed Point Theory Appl. 14, 457-501 (2013)

20. Tanaka, K.: A note on generalized solutions of singular Hamiltonian systems. Proc. Am. Math. Soc. 122, 275-284 (1994)

21. Whittaker, E.T.: A Treatise on the Analytical Dynamics of Particles and Rigid Bodies: With An Introduction to the Problem of Three Bodies, 4th edn. Cambridge University Press, New York (1959)

22. Yu, G.: Periodic solutions of the planar $N$-center problem with topological constraints. Discrete Contin. Dyn. Syst. 36, 5131-5162 (2016) 